\title{
Celiac disease associated liver dysfunction: Study of the histological features and co-localisation patterns of IgA and IgG anti-tissue transglutaminase antibody (TTG-2) in liver biopsies
}

\author{
Rimlee DUTTA ${ }^{* 1}$, Prasenjit DAS', Siddhartha DATTAGUPTA', Govind MAKHARIA ${ }^{2}$ \\ 'Department of Pathology, All India Institute of Medical Sciences, India \\ ${ }^{2}$ Department of Gastroenterology and Human Nutrition, All India Institute of Medical Sciences, India
}

Introduction: Celiac disease (CeD) associated liver dysfunctions (CALD) have wide range of manifestation ranging from milder symptoms alleviating on gluten free diet (GFD), to pathological conditions such as autoimmune hepatitis, cirrhosis etc. However, characteristic histopathological signatures diagnostic of this entity have not been described yet. Diagnosis relies solely on clinical suspicion due to co-occurrence of two pathologies. The aim of this study is to elucidate histological features of CALD and demonstrate $\operatorname{IgA} / \operatorname{IgG}$ anti-tTG colocalization patterns as a hypothetic evidence towards etiology.

Methods: 28 formalin fixed paraffin embedded (FFPE) core liver biopsies from patients with treatment naïve CeD with associated liver dysfunction and another 5 FFPE liver core biopsies from patients with other proven pathologies and normal serum anti-tTG titres, were retrieved and IgA/IgG anti-tTG colocalization was carried out both by dual immunohistochemistry (IHC) and dual confocal immunofluorescence (IF) techniques. After a period of GFD, clinically indicated liver biopsies from 5 of these 28 patients were obtained and subjected to repeat testing via the same techniques.

Results: Histopathological examination in CALD revealed the following spectrum: steatohepatitis 4 (14.2\%), autoimmune hepatitis 4 (14.2\%), non-cirrhotic portal venopathy 1 (3.5\%), secondary hemochromatosis 1 (3.5\%), steatohepatitis with necrotising granuloma 1 (3.5\%), cirrhosis 3 (10.7\%), irregular sinusoidal dilatation 14 (50\%). In CALD, IgA/IgG anti-tTG colocalization was noted in 85.6\% cases by dual IHC technique and in $92.8 \%$ cases by dual IF technique. Follow up biopsies in $5 \mathrm{CeD}$ cases showed no co-localisation.

Conclusions: Deposition of the circulating IgA/IgG anti-tTG antibodies in suspected patients of CALD seems to be a sensitive and specific technique for diagnosing their celiac association, thereby opening up newer insights into management protocols. 